\title{
A HARDY-LITTLEWOOD MAXIMAL INEQUALITY FOR JACOBI TYPE HYPERGROUPS
}

\author{
WILLIAM C. CONNETT AND ALAN L. SCHWARTZ
}

(Communicated by J. Marshall Ash)

\begin{abstract}
A Hardy-Littlewood maximal inequality is proved for a class of probability preserving measure algebras on compact intervals.
\end{abstract}

\section{INTRODUCTION}

The classical maximal average function of Hardy and Littlewood [6] is defined for $f$ periodic with period $2 \pi$ by

$$
\mathscr{M} f(s)=\sup _{\varepsilon>0}\left|\frac{1}{\varepsilon} \int_{s}^{s+\varepsilon} f(t) d t\right| .
$$

Since periodic functions have a convolution $*$ defined by

$$
(f * g)(s)=\int_{-\pi}^{\pi} f(t) g(s-t) d t
$$

an equivalent expression for $(1.1)$ is

$$
\mathscr{M} f=\sup _{\varepsilon>0}\left|\left(k_{\varepsilon} * f\right)(s)\right|
$$

where $k_{\varepsilon}(s)=1 / \varepsilon \quad(0 \leq s \leq \varepsilon)$ and $k_{\varepsilon}(s)=0$ otherwise. The operator satisfies a variety of inequalities: in particular

$$
\|\mathscr{K} f\|_{p} \leq A_{p}\|f\|_{p} \quad(1<p \leq \infty)
$$

where $A_{p}$ is independent of $f ; \mathscr{M}$ is also of weak type $1-1$.

If $m$ is a nonnegative measure on an interval $H$, then (1.1) has a natural generalization to

$$
\mathscr{M} f(s)=\sup _{\varepsilon>0}\left|\frac{1}{m\left(I_{\varepsilon, s}\right)} \int_{I_{c, s}} f(t) d m(t)\right|
$$

Received by the editors June 13, 1988 and, in revised form, September 28, 1988.

1980 Mathematics Subject Classification (1985 Revision). Primary 43A10.

Key words and phrases. Measure algebra, maximal function, hypergroup. 
where $I_{\varepsilon, s}=\{t: t \in H,|s-t| \leq \varepsilon\}$; under some restrictions on $m$, (1.3) holds [1, p. 71]. In many such cases, most notably where $m$ is the measure of orthogonality for the ultraspherical or, more generally, Jacobi polynomials, $L^{1}(\mathrm{dm})$ is a Banach algebra with a multiplicative operator * and in those cases, an analogue of (1.2) also defines a maximal operator which satisfies (1.3); this is implicit in the comparison of the two types of maximal functions established for the ultraspherical case in [2, Lemma, p. 63] and the Jacobi case [3, §3]. Both arguments, especially the latter, involve detailed knowledge of the ultraspherical or Jacobi polynomials and related functions and thus resist generalization. It is apparent in [2, 3], and Theorem 3 (below) that the maximal function analogous to (1.2) has greater utility than that analogous to (1.4) in the delicate analysis of the ultraspherical and Jacobi series.

The aim of this article is to establish analogues to (1.3) for Jacobi type hypergroups, a large class of measure algebras which generalize the Jacobi and ultraspherical examples. So, throughout this paper let $H=[0, \pi]$ and let $(H, *)$ be a Jacobi type $(\alpha, \beta)$ hypergroup. The reader is referred to [5] for precise definitions, examples, and a more complete and detailed discussion of the properties of Jacobi type hypergroups. We cite in this article, as briefly as possible, only those properties actually required for the discussion. For the sake of simplicity in exposition, we assume $\alpha \geq \beta \geq 0$ (the first inequality must necessarily hold in a Jacobi type hypergroup; the second can be replaced by $\beta \geq-1 / 2$ with some modification (see $[5, \S 4.8])$ ).

Under these assumptions $M(H)$ is a commutative Banach algebra with convolution $*$ and identity $\delta_{0}$ where $\delta_{s}$ is the unit point mass concentrated at s. $M(H)$ has a nonnegative measure $m$ with unit total variation such that $m * \delta_{s}=\delta_{s} * m=m$ for every $s \in H$. We use $\|\mu\|$ for the total variation of $\mu \in M(H)$ and $\|f\|_{p}$ for the norm of $f \in L^{p}(d m) ; m$ is called normalized Haar measure and is absolutely continuous with respect to Lebesgue measure.

The operators $T^{s}$ defined by $T^{s} f(t)=\int_{H} f d\left(\delta_{s} * \delta_{t}\right)$ are called generalized translations because they satisfy the definition formulated in [7]. Convolution of functions is defined by

$$
(f * g)(s)=\int_{H} T^{s} f(t) g(t) d m(t)
$$

\section{MAXIMAL OPERATORS ON HYPERGROUPS}

It is now possible to define the objects analogous to (1.1) and (1.2). For $s \in H$ and $\varepsilon>0$ let $I_{\varepsilon, s}=\{t \in H:|t-s| \leq \varepsilon\}, k_{\varepsilon, s}(t)=\left[m\left(I_{\varepsilon, s}\right)\right]^{-1}\left(t \in I_{\varepsilon, s}\right)$ and $k_{\varepsilon, s}(t)=0 \quad\left(t \notin I_{\varepsilon, s}\right)$. Define also $I_{\varepsilon}=I_{\varepsilon, 0}=[0, \varepsilon]$ and $k_{\varepsilon}=k_{\varepsilon, 0}$. The two analogues to the maximal operators of (1.1) and (1.2) are 


$$
\begin{gathered}
M f(s)=\sup _{\varepsilon>0}\left|\int_{I_{\varepsilon, s}} k_{\varepsilon, s}(t) f(t) d m(t)\right|, \\
\mathscr{M} f(s)=\sup _{\varepsilon>0}\left|\left(k_{\varepsilon} * f\right)(s)\right|=\sup _{\varepsilon>0}\left|\int_{I_{\varepsilon, s}} T^{s} k_{\varepsilon}(t) f(t) d m(t)\right| .
\end{gathered}
$$

The operators $M$ and $\mathscr{M}$ are distinct. Indeed, a comparison of the two requires a careful investigation of the relation between the two functions $T^{s} k_{\varepsilon}$ and $k_{\varepsilon, s}$; in the case of Jacobi expansions the comparison requires knowledge of the exact form of the convolution kernel [3, Theorem 4]. There is no apparent path to a comparison of the two for Jacobi type hypergroups; nevertheless, it is still possible to obtain an analogue to some of the Hardy and Littlewood results.

Theorem 1. There are constants $A_{p}$ such that

$$
\|M f\|_{p} \leq A_{p}\|f\|_{p} \quad(1<p \leq \infty)
$$

moreover, $M$ is also of weak type $1-1$.

Proof. The result follows easily from [1, p. 71] since from the theory of Jacobi type hypergroups [5, Theorem 4.4 and Lemma 3.1] it is known that $\operatorname{dm}(t)=$ $\rho^{2}(t) d t$ where $\rho$ is positive on $(0, \pi)$ and there are positive real numbers $a_{\alpha}$ and $a_{\beta}$ such that

$$
\lim _{t \rightarrow 0+} t^{-\alpha-1 / 2} \rho(t)=a_{\alpha} \text { and } \lim _{t \rightarrow \pi-}(\pi-t)^{-\beta-1 / 2} \rho(t)=a_{\beta} .
$$

This implies that $[0, \pi]$ with the measure $m$ is of "homogeneous type."

Theorem 2. There are constants $A_{p}$ such that

$$
\|\mathscr{M} f\|_{p} \leq A_{p}\|f\|_{p} \quad(1<p \leq \infty)
$$

Theorem 2 generalizes results for Jacobi expansions [3, Theorem 8], but utilizes a different approach first formulated in [4]. The proof of Theorem 2 requires some additional definitions and facts about Jacobi type hypergroups.

A function $\varphi$ in $C(H)$ which satisfies $\int_{H} \varphi d\left(\delta_{s} * \delta_{t}\right)=\varphi(s) \varphi(t)$ for every $s, t \in H$ is called a character of $(H, *)$. The set of characters is countable and constitutes a complete orthogonal system for $L^{2}(\mathrm{dm})$. Corresponding to each character $\varphi$ is a nonnegative real number $\lambda$ such that

$$
\left(\rho^{2} \varphi^{\prime}\right)^{\prime}+\lambda^{2} \rho^{2} \varphi=0 \text {. }
$$

The characters can be numbered $\varphi_{0}, \varphi_{1}, \varphi_{2}, \cdots$ so that the corresponding values of $\lambda$ form an increasing sequence $\lambda_{0}, \lambda_{1}, \lambda_{2}, \cdots$. Define $f^{\wedge}(k)=$ $\int_{H} f \varphi_{k} d m$ and $h_{k}=\left\|\varphi_{k}\right\|_{2}^{-2}$ then

$$
\sum_{k=0}^{\infty}\left|f^{\wedge}(k)\right|^{2} h_{k}=\int_{H}|f|^{2} d m .
$$


In addition

$$
\begin{gathered}
\left\|\varphi_{k}\right\|_{\infty}=1, \\
\lim _{k \rightarrow \infty} k^{-1} \lambda_{k}=1, \\
\lim _{k \rightarrow \infty} k^{2 \alpha+1} h_{k}=a>0,
\end{gathered}
$$

so the following analogue of the Poisson kernel is well defined:

$$
P_{r}(t)=\sum_{k=0}^{\infty} \exp \left(-\lambda_{k} r\right) h_{k} \varphi_{k}(t)
$$

as is the corresponding maximal function:

$$
f^{*}(t)=\sup _{r>0}\left|\left(P_{r} * f\right)(t)\right|
$$

Proof of Theorem 2. The argument proceeds in three steps. First it will be shown that there are constants $C_{p}$ such that

$$
\left\|f^{*}\right\|_{p} \leq C_{p}\|f\|_{p} \quad(1<p \leq \infty) .
$$

Second, it will be shown that there are positive constants $E$ and $G$ such that

$$
k_{\varepsilon}(t) \leq E P_{G \varepsilon}(t) \quad(t \in H, \varepsilon>0) \text {. }
$$

Finally (2.7) implies that $\left(k_{\varepsilon} * f\right)(t) \leq E\left(P_{G \varepsilon} * f\right)(t) \leq E f^{*}(t)$, so that $\mathscr{M} f(t) \leq E f^{*}(t)$ and the theorem follows from (2.6) with $A_{p}=E C_{p}$.

Step 1. Let $W_{r}(t)=\sum_{k=0}^{\infty} \exp \left(-\lambda_{k}^{2} r\right) h_{k} \varphi_{k}(t)$, so that if $f \in L^{1}(d m)$ and $\sum\left|f^{\wedge}(k)\right| h_{k}<\infty$, then separation of variables together with (2.2) shows that $u(r, t)=\left(W_{r} * f\right)(t)$ satisfies $u(0, t)=f(t)$ and $\left(\rho^{2}(t) u_{t}\right)_{t}-\rho^{2}(t) u_{r}=0$. If $f(t) \geq 0$ for every $t$ in $H$, then the maximum principle for parabolic equations [8] guarantees that $u(r, t) \geq 0(r>0,0<t<\pi)$, hence $W_{r} \geq 0$ on $H$, and so by a subordination argument, (see $[9$, pp. 47-48])

$$
P_{r}(t)=\pi^{-1 / 2} \int_{0}^{\infty} v^{-1 / 2} e^{-v} W_{r^{2} / 4 v} d v \geq 0 .
$$

If $S^{r} f=P_{r} * f$, then $\left\{S^{r}\right\}$ is a symmetric diffusion semigroup in the sense of $\left[9\right.$, p. 65]. For instance, to see that $\left\{S^{r}\right\}$ satisfies the contraction property, let $1 \leq p \leq \infty$ and let $q$ be the conjugate index to $p$. Then if $f \in L^{p}(d m)$ and $g \in L^{q}(d m)$

$$
\left|\int_{0}^{\pi} S^{r} f(t) g(t) d m(t)\right|=\left|\int_{0}^{\pi} \int_{0}^{\pi} T^{t} P_{r}(s) f(s) g(t) d m(s) d m(t)\right| .
$$

Now $P_{r}(s) \geq 0$ in $H$, so $T^{t} P_{r}(s) \geq 0$ on $H \times H$. Hence $T^{t} P_{r}(s) d m(s) d m(t)$ defines a nonnegative measure on $H \times H$ of unit total variation. If Hölder's inequality with this measure is invoked in the last integral, then Fubini's Theorem together with the observation that $T^{t} P_{r}(s)=T^{s} P_{r}(t)$ may be used to obtain the 
bound $\|f\|_{p}\|g\|_{q}$ thus $\left\|S^{r} f g\right\|_{1} \leq\|f\|_{p}\|g\|_{q}$, whence $\left\|S^{r} f\right\|_{p} \leq\|f\|_{p}$ which is the contraction property. Equation (2.6) is now equivalent to Stein's maximal theorem [9, p. 73].

Step 2. Equations (2.4) and (2.5) ensure the existence of positive constants $K, C_{1}, C_{2}, C_{3}$ and $C_{4}$ such that for $k=0,1,2, \cdots$

$$
C_{1} k \leq \lambda_{k} \leq C_{2} k, \quad C_{3} k^{2 \alpha+1} \leq h_{k} \leq C_{4} k^{2 \alpha+1} .
$$

Also $\varphi_{k}(t) \geq 3^{-1} \quad(k t \leq K)$ [5, Theorem 4.3]. Since $k_{\varepsilon}(t)=0$ for $t>\varepsilon$ and $P_{\varepsilon}(t) \geq 0$, it suffices to consider $t<\varepsilon$. Let

$$
\begin{aligned}
& E_{1}=\{k: 0 \leq k \leq K / \varepsilon\}, \\
& E_{2}=\{k: K / \varepsilon<k \leq K / t\}, \\
& E_{3}=\{k: k>K / t\},
\end{aligned}
$$

and $\Sigma_{j}=\sum_{k \in E_{j}} \exp \left(-\lambda_{k} \varepsilon\right) h_{k} \varphi_{k}(t)$.

If $k \in E_{1}, \lambda_{k} \varepsilon \leq C_{2} k \varepsilon \leq C_{2} K$ and $k t<k \varepsilon<K$, then

$$
\Sigma_{1} \geq 3^{-1} \exp \left(-C_{2} K\right) \sum_{k \in E_{1}} h_{k}
$$

and

$$
\sum_{k \in E_{1}} h_{k} \geq C_{3}(2 \alpha+2)^{-1}(K / \varepsilon)^{2 \alpha+2}
$$

whence for some constant $C_{5}>0, \Sigma_{1} \geq C_{5} \varepsilon^{-2(x-2}$. For $k \in E_{2}, \varphi_{k}(t) \geq 1 / 3$ so $\Sigma_{2} \geq 0$.

Let $G>1$ and assume $G t<\varepsilon$ and $k \in E_{3}$, then $k>K / t>K G / \varepsilon$ and by (2.3)

$$
\left|\Sigma_{3}\right| \leq \sum_{k>K G / \varepsilon} \exp \left(-C_{1} k \varepsilon\right) C_{4} k^{2(\alpha+1}
$$

hence for some constant $C_{6}>0$

$$
\begin{aligned}
\left|\Sigma_{3}\right| & \leq C_{6} \int_{K G / \varepsilon}^{\infty} \exp \left(-C_{1} x \varepsilon\right) x^{2 \alpha+1} d x \\
& =C_{6} \varepsilon^{-2 \alpha-2} \int_{K G}^{\infty} \exp \left(-C_{1} y\right) y^{2(x+1} d y,
\end{aligned}
$$

so if $G$ is sufficiently large $\left|\Sigma_{3}\right| \leq \Sigma_{1} / 2$, so

$$
P_{\varepsilon}(t)=\Sigma_{1}+\Sigma_{2}+\Sigma_{3} \geq \frac{1}{2} C_{5} \varepsilon^{-2(x-2} \quad(0 \leq t<\varepsilon / G) .
$$

Because of (2.1) there is a constant $C_{7}$ such that $C_{7} \rho^{2}(t) \geq t^{2 \alpha+1}>0$ so

$$
k_{\varepsilon}(t)=\left[\int_{0}^{\varepsilon} d m(s)\right]^{-1} \leq C_{7}(2 \alpha+2) \varepsilon^{-2 \kappa-2} .
$$

Since $G$ is independent of $\varepsilon$ there is a constant $C$ such that

$$
k_{\varepsilon / G}(t) \leq k_{\varepsilon}(t) \leq C P_{\varepsilon}(t) \quad(0 \leq t<\varepsilon / G)
$$

which is equivalent to $(2.7)$. 
Many of the problems of harmonic analysis require for their solutions information about some parametrized family of convolution operators. Examples include convolution with the kernels $k_{\varepsilon}, P_{r}$ and $Q_{r}$ (defined below). Summability methods such as Cesaro summability inevitably lead to similar operators where the parameter usually runs through the positive integers. The following theorem generalizes the observation of Hardy and Littlewood that such operators can be controlled by $\mathscr{M}$.

Theorem 3. Let $w_{p}(t)$ be an integrable function that depends on a parameter $p$ and define $h(p, t)=\left(w_{p} * f\right)(t)$. Assume either

(a) there is $B>0$, independent of $p$ such that

$$
\left|\int_{0}^{\pi} w_{p}(t) d m(t)\right| \leq B, \quad \int_{0}^{\pi}\left|m[(0, t)] \frac{d}{d t} w_{p}(t)\right| d t \leq B,
$$

or

(b) there are functions $W_{p}(t) \geq\left|w_{p}(t)\right|$ and $C$ independent of $p$ such that $W_{p}(\pi) \leq C$ and

$$
\int_{0}^{\pi}\left|m[(0, t)] \frac{d}{d t} W_{p}(t)\right| d t \leq C
$$

then there is $K$ independent of $p$ such that

$$
|h(p, t)| \leq K \mathscr{M} f(t) .
$$

The proof of Theorem 2 is very similar to that of [3, Theorem 5].

Remarks. It is interesting to note the contrast between the argument for Theorem 2 and the proof of the corresponding results in [ 3 and 6]. Theorem 2 is the product of inequalities for $f^{*}$ and the inequality $\mathscr{M} f(t) \leq C f^{*}(t)$. The approach in [3], which is valid only for Jacobi expansions, first uses the Coifman and Weiss result $[1$, p. 71$]$ to assert that $M$ is a bounded operator on $L^{p}(d m)$ $(1<p \leq \infty)$; then the detailed knowledge of the convolution kernel for Jacobi expansions is used to show that $M$ dominates $\mathscr{M}$. Finally, Theorem 3 can be used to obtain bounds for the operator $P_{*} f(t)=\sup _{r>0}\left|\left(Q_{r} * f\right)(t)\right|$ where

$$
Q_{r}(t)=\sum_{k=0}^{\infty} e^{-k r} h_{k} \varphi_{k}(t) .
$$

This is possible because an explicit closed form is known for $Q_{r}(t)$. (See $[3,(2.2)]$ and the reference cited there.) The scheme followed in this paper first establishes inequalities for $f^{*}$ (which in the light of $(2.4)$ is an object very like $\left.P_{*} f\right)$ and then uses $f^{*}$ to dominate $\mathscr{M} f$.

The methods of [3] have, as a reward for detailed computation in a special case, the bonus of weak type $1-1$ results and, for all the bounds obtained, a wider range of $(\alpha, \beta)$ than would be obtained by specializing Theorem 2 to Jacobi expansions. 


\section{ACKNOWLEDGMENT}

The second author wishes to express his gratitude to the School of Mathematical Sciences at Tel-Aviv University for its hospitality during the preparation of this paper.

\section{REFERENCES}

1. R. Coifman and G. Weiss, Analyse harmonique non-commutative sur certains espaces homogenes, Lecture Notes in Mathematics, vol. 242, Springer-Verlag, Berlin and New York.

2. W. C. Connett and A. L. Schwartz, The theory of ultraspherical multipliers, Mem. Amer. Math. Soc., vol. 9, no. 183, 1977.

3. _ The Littlewood-Paley theory for Jacobi expansions, Trans. Amer. Math. Soc. 251 (1979), 219-234.

4. __ The harmonic machinery for eigenfunction expansions, Proc. Sympos. Pure Math., vol. 35, Amer. Math. Soc., Providence, R.I., pp. 429-434.

5. __ Analysis of a class of probability preserving measure algebras on a compact interval, Trans. Amer. Math. Soc. (to appear).

6. G. H. Hardy and J. E. Littlewood, A maximal theorem with function theoretic applications, Acta Math. 54 (1930), 81-116.

7. B. M. Levitan, Generalized translation operators, Israel Program for Scientific Translations, Jerusalem, 1964.

8. M. H. Protter and H. F. Weinberger, Maximum principles in differential equations, PrenticeHall, Englewood Cliffs, N.J., 1967.

9. E. M. Stein, Topics of harmonic analysis related to the Littlewood-Paley Theory, Ann. of Math. Stud., no. 63, Princeton Univ. Press, Princeton, N.J., 1970.

Department of Mathematics, University of Missouri, St. Louis, Missouri 63121 